\title{
Variación espacio-temporal de macroinvertebrados acuáticos en la Lindosa, Guayana colombiana
}

\author{
Gabriela Córdoba-Ariza ${ }^{1}$, Katterine Rincón-Palau ${ }^{1}$, Jhon Ch. Donato-R. ${ }^{1}$ \& \\ Juan David González-Trujillo ${ }^{1,2}$ \\ 1. Departamento de Biología, Universidad Nacional de Colombia, Cra. 30 No. 45-01, Bogotá, D.C., Colombia; \\ pgcordobaa@unal.edu.co, kjrinconp@unal.edu.co, jcdonator@unal.edu.co \\ 2. Instituto Catalán de Investigaciones del Agua (ICRA), Carrer Emili Grahit 101, 17003 Girona, España; \\ jdgonzalezt@unal.edu.co
}

\section{Recibido 15-X-2019. Corregido 31-XII-2019. Aceptado 04-III-2020.}

\begin{abstract}
Spatio-temporal variation of the aquatic macroinvertebrates in La Lindosa, Colombian Guiana. Introduction: Describing biodiversity patterns and the underlying drivers is relevant for tackling the rampant pressures on tropical freshwaters. This is particularly relevant in scarcely studied regions, such as in the Colombian Guiana shield. Objective: To describe the taxonomic and functional structure of macroinvertebrate communities and assess their variability across spatial and temporal scales. Methods: We sampled the invertebrate communities in four streams during the dry seasons of 2017 and 2018. Additionally, we assessed diversity patterns within and among streams and between different sampling months in one stream. To assess diversity patterns, we compared alpha (differences in taxon richness and abundance within communities) and beta (differences in taxon composition among communities) diversity patterns among and within rivers. Alpha diversity was estimated using the numbers of Hill, while beta diversity was estimated using the Sørensen and Morisita-Horn indices and compared using a similarities analysis (ANOSIM). Results: The taxon composition of the communities was different within (mesohabitat scale) and among streams (regional scale). However, the composition of FFGs changed among but not within streams. The comparison of the numbers of Hill and the taxon composition suggested that the mesohabitat type is a determining factor of the taxonomic but not FFG diversity across scales. At the temporal scale, alpha diversity was low for months with high precipitation (February-April) and high for the dry season (January). The temporal beta diversity was high among sampling periods, being nestedness processes driving beta diversity among years and turnover processes driving beta diversity within years. Conclusion: Streams from Serranía de la Lindosa host a high diversity of freshwater macroinvertebrates. Seasonality and differences in the in-stream habitat heterogeneity seem to determine the diversity patterns observed at different temporal and spatial scales. A deeper study is needed to understand the functioning of these ecosystems and improve the ability to generate management and conservation strategies.
\end{abstract}

Key words: regional diversity, habitat features, functional feeding group, scale, Orinoco, Neotropical.

Córdoba-Ariza, G., Rincón-Palau, K., Donato-R. J.Ch., \& González-Trujillo, J.D. (2020). Variación espacio-temporal de macroinvertebrados acuáticos en la Lindosa, Guayana colombiana. Revista de Biología Tropical, 68(2), 452-465.

El escudo Guayanés se compone de alrededor de 2.5 millones de $\mathrm{km}^{2}$ que se ubican al norte de Suramérica entre Venezuela, Guyana, Surinam, Guayana Francesa, Colombia y Brasil (Hammond, 2005). Dada su ubicación geográfica, en la que confluyen tanto elementos amazónicos como orinocenses y andinos, y sus características ambientales únicas, es una región muy biodiversa en la que se registra un alto número de endemismos (e.g. Giraldo-Cañas, 2001, Salerno et al., 2012). Sin embargo, en la actualidad, es también una 
región en la cual gran parte de esta biodiversidad, y de los factores que la determinan, es aún desconocida.

Dentro de los estudios llevados a cabo en la región del escudo guyanés destacan algunas caracterizaciones de vegetación (Giraldo-Cañas, 2001; Cárdenas López, Castaño Arboleda, Zubieta Vega, \& Jaramillo Echeverry, 2008), fauna y algunos grupos de algas y macrófitas (e.g. Lasso \& Morales-Betancourt, 2017; Lasso, Morales-Betancourt, \& EscobarMartínez, 2019; Atuesta-Ibargüen, 2019). En el caso particular de los macroinvertebrados acuáticos, su diversidad sólo ha sido descrita en ríos de la Serranía de la Macarena (GranadosMartínez \& Batista, 2017; Granados-Martínez, Lasso, \& Núñez-Avellaneda, Marcela MoralesBetancourt, 2018) y en el río Bita (GranadosMartínez \& Montoya, 2017).

Si bien caracterizar la diversidad mediante listados de especies es un paso esencial, es importante considerar que los patrones de diversidad no son estáticos, sino que, por el contrario, varían dependiendo de la acción de factores actuando en distintas escalas espaciales y temporales (Ligeiro, Melo, \& Callisto, 2010; Gabbud, Robinson \& Lane, 2019). Por tanto, caracterizar la diversidad y su variación en el tiempo y espacio es de gran relevancia en aras de la conservación de zonas de gran biodiversidad, como el escudo guyanés. Teniendo en cuenta este panorama, este trabajo tiene como objetivo describir la estructura taxonómica y funcional de las comunidades de macroinvertebrados de la Serranía la Lindosa (Colombia), evaluando su variación a lo largo de escalas espaciales y temporales.

\section{MATERIALES Y MÉTODOS}

Área de estudio: Los arroyos estudiados fueron La Lindosa $\left(2^{\circ} 30^{\prime} \mathrm{N} \& 72^{\circ} 38^{\prime} \mathrm{W}\right)$, Tranquilandia ( $2^{\circ} 30^{\prime} \mathrm{N} \& 72^{\circ} 42^{\prime}$ W), el Rincón de los Toros ( $2^{\circ} 31^{\prime} \mathrm{N} \& 72^{\circ} 41^{\prime}$ 'W) y Retirito $\left(2^{\circ} 29^{\prime} \mathrm{N} \& 72^{\circ} 43^{\prime} \mathrm{W}\right)$ todos ubicados en la Serranía de La Lindosa (Fig. 1). Esta formación rocosa de aproximadamente 12000 ha se encuentra en el departamento del Guaviare, cerca al municipio de San José del Guaviare, y forma parte de la zona norte de la amazonia colombiana, en la transición con la región de la Orinoquía (Cárdenas López et al., 2008). En general, el clima se cataloga como tropical lluvioso y la precipitación tiene un régimen

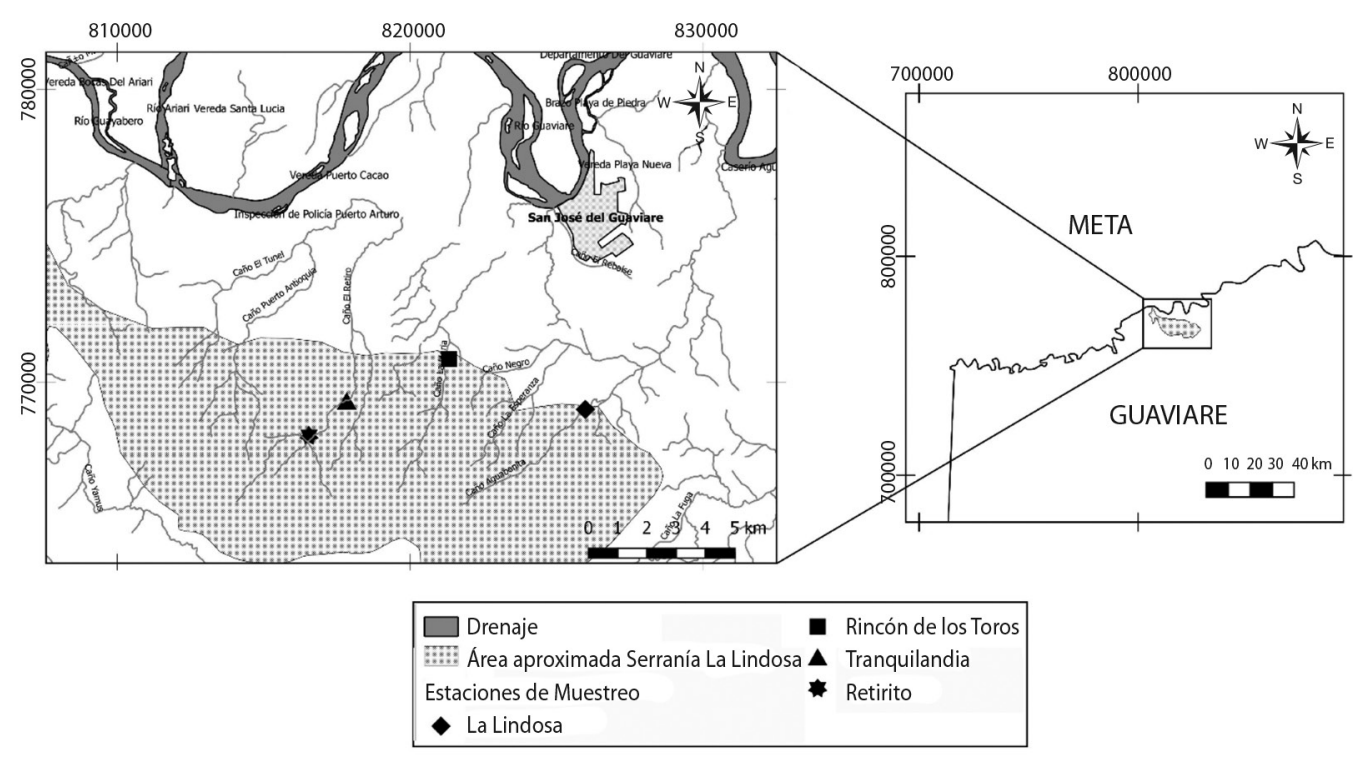

Fig. 1. Serranía de La Lindosa (San José De Guaviare, Colombia). Ubicación de los cuatro arroyos estudiados.

Fig. 1. Serranía de La Lindosa (San José De Guaviare, Colombia). Location of the four studied streams. 
monomodal con un promedio de $2800 \mathrm{~mm}$ al año; el período lluvioso va de marzo a octubre y el seco de noviembre a febrero (Cárdenas López et al., 2008).

Los arroyos de formaciones como $\mathrm{La}$ Lindosa se caracterizan por tener lechos compuestos por grandes losas que corresponden a relictos del escudo guyanés y secuencias marcadas de rápidos y pozos cuyas transiciones están dadas frecuentemente por cascadas de altura variable. A lo largo del cauce pueden encontrarse un gran número de especies de macrófitas dentro de las cuales Rhyncholacis clavigera (P. Royen) es de las más representativas. En cuanto a los aspectos físico-químicos (Tabla 1) los arroyos de la Guayana pueden ser considerados como ultra-oligotróficos. Esto se refleja en que el agua que los recorre tiene una baja concentración y disponibilidad de nutrientes y valores de conductividad cercanos a los
$2 \mu \mathrm{S} / \mathrm{cm}^{3}$. Adicionalmente, se caracterizan por registrar algunos de los pHs más ácidos en todo el neotrópico con rangos que oscilan entre $4.5 \mathrm{y}$ 6.0 (Hammond, 2005).

\section{Muestreo y procesamiento de las mues-} tras: En enero de 2017, se muestrearon los afluentes de La Lindosa, Tranquilandia y el Rincón de los Toros y en enero del 2018, se llevó a cabo el muestreo del arroyo El Retirito. En cada afluente se estableció un tramo representativo (150-200 m) que contuviera la mayor diversidad de sustratos observados a lo largo de todo el afluente: roca madre $(>100 \mathrm{~cm})$, bloques $(25-100 \mathrm{~cm})$, cantos $(6-25 \mathrm{~cm})$, guijarros $(2-6 \mathrm{~cm})$, gravas $(0.2-2 \mathrm{~cm})$, macrófitas $(R$. clavigera) y hojarasca. Posteriormente, se realizó un muestreo multihábitat según la metodología propuesta por Elosegi y Sabater (2009) utilizando una red Surber de $0,09 \mathrm{~m}^{2}$ de diámetro

TABLA 1

Valores promedio de variables geomorfológicas, hidrológicas, físicas y químicas de los cuatro arroyos de la Serranía de La Lindosa medidas durante la época seca del 2017 y del 2018

TABLE 1

Average values of geomorphological, hydrological, physical and chemical variables in the four streams of the Serranía de La Lindosa measured during the dry season of 2017 and 2018

\begin{tabular}{|c|c|c|c|c|c|}
\hline Componente & Variables & La Lindosa & R. de los Toros & Tranquilandia & Retirito \\
\hline \multirow[t]{2}{*}{ Geomorfológico } & Elevación (m.s.n.m) & 208.0 & 228.0 & 227.0 & 273.0 \\
\hline & Pendiente del tramo $\left({ }^{\circ}\right)$ & 2.6 & 2.7 & 2.7 & 2.0 \\
\hline \multirow[t]{5}{*}{ Hidrológico } & Caudal promedio $\left(\mathrm{m}^{3} / \mathrm{s}\right)$ & $0.28(0.05)$ & $0.26(0.05)$ & $0.29(0.09)$ & 0.36 \\
\hline & Profundidad promedio (m) & $0.11(0.02)$ & $0.12(0.04)$ & $0.10(0.04)$ & $0.58(0.06)$ \\
\hline & Ancho promedio (m) & $9.2(2.2)$ & $10.4(1.4)$ & $10.1(3.2)$ & 13.6 \\
\hline & Solidos disueltos totales (mg/l) & 0.0478 & 0.0016 & 0.0160 & 1.000 \\
\hline & Solidos suspendidos (mg/l) & 0.0043 & 0.0001 & 0.0005 & - \\
\hline \multirow[t]{6}{*}{ Físico } & Coeficiente de extinción de la luz (k) & 18.0 & 15.6 & 9.8 & 1.7 \\
\hline & Temperatura promedio $\left({ }^{\circ} \mathrm{C}\right)$ & $27.7(1.2)$ & $26.1(0.2)$ & $25.4(0.1)$ & $25.3(0.5)$ \\
\hline & Conductividad promedio (uS/cm) & $3.8(0.2)$ & $2.4(0.4)$ & $3.1(0.2)$ & $2.5(0.71)$ \\
\hline & $\mathrm{pH}$ & $5.9(0.1)$ & $5.2(0.2)$ & $5.8(0.5)$ & $5.3(0.01)$ \\
\hline & Oxígeno disuelto promedio (mg/l) & $7.1(0.3)$ & $7.2(0.2)$ & $7.2(0.2)$ & $2.2(0.1)$ \\
\hline & Porcentaje de saturación de $\mathrm{O} 2$ & 89.8 & 89.5 & 91.4 & 28.1 \\
\hline \multirow[t]{5}{*}{ Químico } & Fósforo reactivo (ug/l PRS) & 5.6 & 5.4 & 5.5 & 16.2 \\
\hline & Amonio (ug/1 N-NH4) & 9.9 & 24.9 & 23.7 & 10.6 \\
\hline & Nitratos $\left(\mathrm{N}-\mathrm{NO}_{3}(\mathrm{mg} \mathrm{N} / \mathrm{l})\right)$ & 0.007 & 0.006 & 0.013 & 0.040 \\
\hline & Materia orgánica total & 3.4 & 5.8 & 2.5 & - \\
\hline & Fosfatos $\left(\mathrm{P}-\mathrm{PO}_{4}(\mathrm{mg} \mathrm{N} / \mathrm{l})\right)$ & 0.001 & 0.001 & 0.001 & - \\
\hline
\end{tabular}

Dentro de los paréntesis se indican las desviaciones estándar. 
y $200 \mu \mathrm{m}$ de abertura de malla. En la campaña del 2017 se tomaron 15 muestras por arroyo y en el 2018, 10 muestras en el arroyo el Retirito, por lo que el esfuerzo de muestreo total correspondió a 55 muestras ( 3 ríos x 15 muestras + 1 río x 10 muestras). Las muestras fueron preservadas de forma individual en etanol al $96 \%$ en frascos de plástico y llevadas al laboratorio para su posterior estudio taxonómico.

Caracterización de la diversidad taxonómica y funcional de los macroinvertebrados de la Lindosa: La identificación de los ejemplares de macroinvertebrados se realizó hasta el nivel de género mediante las claves de Domínguez y Fernández (2009) y Merritt, Cummins y Berg (2008). Los individuos de la familia Chironomidae fueron aclarados en KOH y montados con Euparal ${ }^{\circledR}$, para ser determinados con la ayuda de las claves de Wiederholm (1983) y Trivinho-Strixino (2011). Se montaron las pupas y exuvias pupales de algunos taxones para corroborar las determinaciones taxonómicas siguiendo las claves de Prat, González-Trujillo y Ospina-Torres (2014).

Luego de su determinación taxonómica, los organismos fueron clasificados en grupos funcionales tróficos (FFG) de acuerdo a la bibliografía. De ser posible, se restringió la información utilizada a estudios realizados en el neotrópico para evitar la incorrecta categorización de los organismos. Para este propósito, se utilizaron los grupos definidos por Cummins (1973) y las traducciones sugeridas en la revisión de Ramírez \& Gutiérrez-Fonseca (2014). Aquellos taxones de los que no se encontró clasificación alguna, fueron etiquetados como "sin información". Por otro lado, aquellos taxones clasificados en más de un FFG se denominaron como de "hábitos variables".

Para determinar la completitud de la comunidad, se utilizaron curvas de cobertura estimadas a partir de una matriz de presencias y ausencias (Chao \& Jost, 2012). La cobertura es una medida que indica la proporción del número total de individuos en una comunidad que fueron capturados en las muestras (Chao \& Jost, 2012). Por otro lado, la diversidad taxonómica se caracterizó mediante la estimación de los tres primeros números de Hill según la propuesta de Chao et al. (2014). Analizar estos tres números en conjunto permite evaluar no sólo la riqueza, entendida como el número efectivo de especies, sino también la dominancia de los diferentes taxones. El primer número de Hill $(\mathrm{q}=0)$ es un equivalente a la riqueza de especies y no le da peso alguno a la abundancia de las especies; el segundo $(\mathrm{q}=1)$ es equivalente al exponencial de la entropía de Shannon y da igual peso a especies dominantes (las de mayor abundancia) y a especies raras; y el tercero $(q=2)$, es equivalente a la diversidad de Simpson y le da un mayor peso a los taxones dominantes.

Variaciones espaciales en la diversidad: Para caracterizar la variación espacial en la comunidad se utilizaron los datos tomados durante enero del 2017 y 2018 (época seca) con el fin de disminuir la posible influencia de la estacionalidad. La variación de la diversidad fue caracterizada en dos escalas espaciales: entre los ríos (i.e. a escala regional) y dentro de los ríos (i.e. a escala de los sustratos, los cuales son considerados como distintos mesohábitats).

Los cambios en la composición taxonómica y de FFG a escala regional y de mesohábitat fueron explorados mediante un escalamiento multidimensional no métrico (NMDS, Kruskal, 1964) y la existencia de diferencias estadísticamente significativas fue evaluada mediante un análisis de similitudes (ANOSIM, Clarke, 1993). En ambos análisis se utilizó una matriz de distancias estimada con el coeficiente de Morisita-Horn (Horn, 1966), ideal para las matrices de datos de conteos biológicos que cuentan con gran número de ceros. Con el objetivo de quitar el peso de los taxones raros y ocasionales, no fueron considerados en el análisis aquellos géneros con un número de individuos menor a 10 .

Variaciones temporales en la diversidad: Para el análisis de la variabilidad temporal en la diversidad se identificaron los individuos provenientes de muestras tomadas en octubre 2014 
y abril y octubre 2015 así como de muestreos mensuales realizados en enero, febrero, marzo, abril y junio 2017 (en total, ocho ocasiones de muestreo). Todas las muestras fueron tomadas en el arroyo Tranquilandia bajo el mismo protocolo de muestreo. Aunque la red Surber utilizada fue la misma $\left(0,09 \mathrm{~m}^{2}\right.$ de diámetro y 200 micras de abertura de malla) el número de muestras tomadas en cada periodo varió, por lo que las abundancias en las muestras fueron ajustadas a la mayor área común $\left(0.45 \mathrm{~m}^{2}\right.$ equivalente al área de 5 muestras de Surber).

Los cambios en la diversidad alfa fueron evaluados en términos de la riqueza y la diversidad de Simpson. Mientras que cambios en la composición fueron evaluados mediante el análisis de la diversidad beta calculada a partir del índice de Sørensen. La diversidad beta es una medida del grado de variación entre dos muestras en términos de la composición de especies. El índice de Sørensen, en particular, evalúa la variación de la composición de especies sin considerar sus abundancias, lo que disminuye el posible efecto de la diferencia en el número total de individuos recolectados en cada campaña de muestreo. Adicionalmente, permite descomponer esta variabilidad en dos componentes que brindan luces a la hora de generar hipótesis sobre los fenómenos ecológicos que subyacen los patrones observados: el anidamiento y el recambio. El anidamiento ocurre cuando las comunidades con menor número de especies son subconjuntos de las más ricas, lo que refleja una pérdida de especies resultado de un proceso no aleatorio; por otra parte, el recambio implica que algunas especies son reemplazadas por otras como consecuencia de filtros ambientales o de limitaciones históricas o espaciales (Baselga, 2010). Todos los análisis descritos fueron realizados en R v3.4.2 utilizando los paquetes "vegan" (Oksanen et al., 2017), "iNEXT" (Chao et al., 2014; Hsieh, Ma, \& Chao, 2016) y "betapart” (Baselga et al., 2018).

\section{RESULTADOS}

Caracterización de la diversidad taxonómica y funcional de los macroinvertebrados de la Lindosa: En total se contabilizaron 13036 individuos distribuidos en 89 géneros pertenecientes a 41 familias y 10 órdenes de invertebrados acuáticos (Apéndice digital 2). En la Tabla 2 se presenta la riqueza de géneros y la abundancia de individuos de cada arroyo. El orden más representativo fue Diptera con 9 familias y 9380 individuos identificados, seguido de Trichoptera (9 familias y 1385 individuos) y Ephemeroptera (6 familias y 1012 individuos). La familia Chironomidae presentó la mayor riqueza (26 géneros) y abundancia (6 685 individuos), seguido en riqueza por la familia Elmidae ( 9 géneros) y en abundancia por la familia Simulidae (2 582 individuos) y Baetidae (727 individuos). Cuatro géneros se registran por primera vez para el país: Luchoelmis (Elmidae), Macropelopia y Phaenopsectra (Chironomidae) y Mayatrichia (Hydroptilidae).

En lo que respecta a los FFG (Tabla 2; Apéndice digital 2, Tabla 1) los depredadores presentaron la mayor riqueza (26 géneros) mientras que la menor riqueza correspondió a los filtradores (3 géneros). Por otro lado, los recolectores fueron los más abundantes (6440 individuos) y los filtradores los menos abundantes (14 individuos). El análisis de completitud indicó una cobertura de más del $90 \%$, lo que indica que el muestreo permitió capturar una gran proporción de los taxones que habitan en los cuatro arroyos (Fig. 1A, Apéndice digital 1). A menor escala, no todos los sustratos alcanzaron coberturas mayores al $80 \%$, siendo la roca madre y las macrófitas los mejor representados (Fig. 1B, Apéndice digital 1).

Variaciones espaciales en la diversidad: Se registraron diferencias en términos de diversidad alfa y composición de especies en las dos 


\section{A}
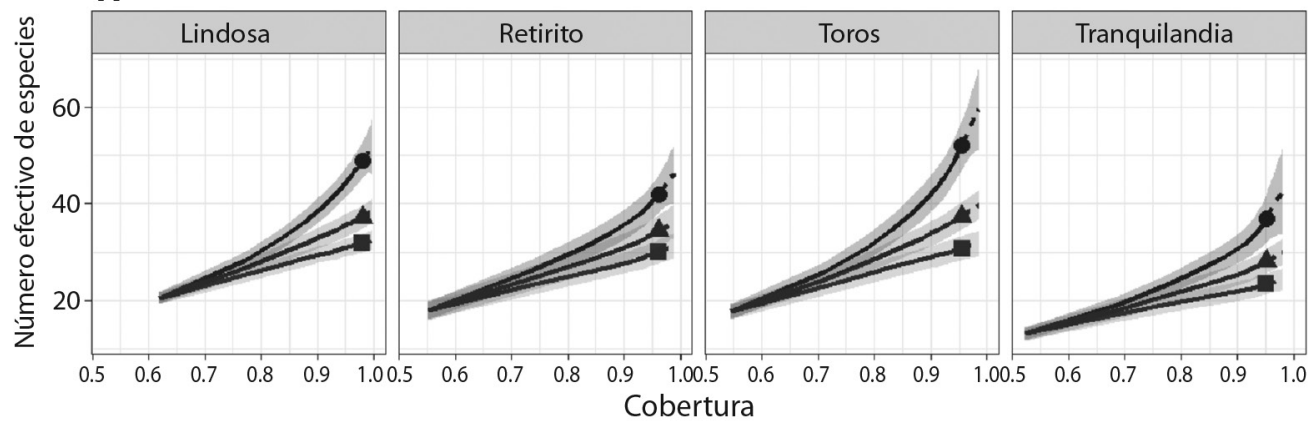

\section{B}
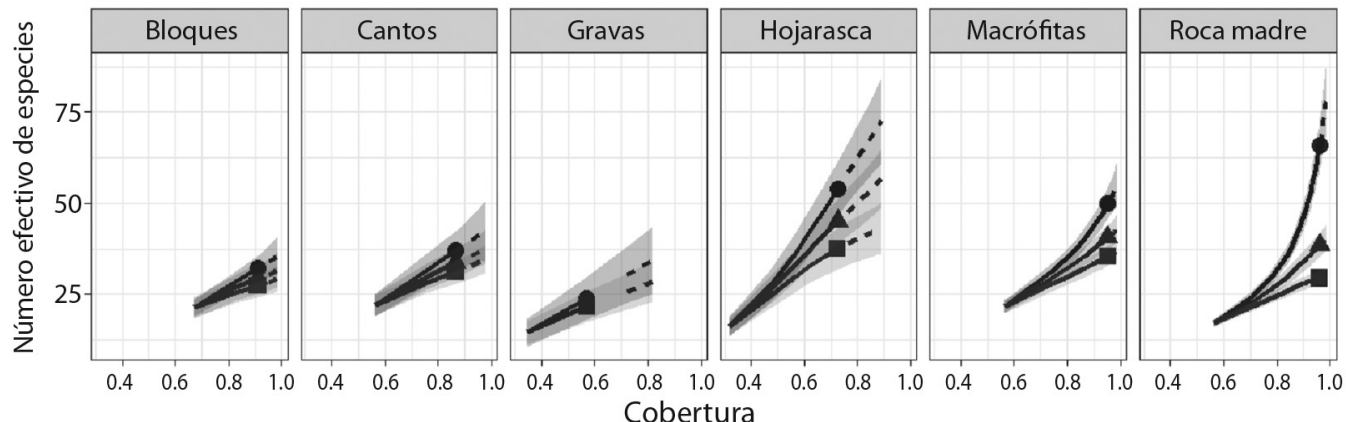

-Interpolado - ' Extrapolado

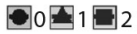

Fig. 2. Estimación del número efectivo de especies como función de la cobertura de acuerdo a los tres primeros números de Hill $(\mathrm{q}=0, \mathrm{q}=1 \mathrm{y} \mathrm{q}=2$ ) para arroyos $(\mathbf{A})$ y mesohábitats $(\mathbf{B})$. Las áreas sombreadas corresponden a intervalos de confianza del $95 \%$.

Fig. 2. Estimation of the effective number of species as a function of the coverage according to the first three numbers of Hill $(q=0, q=1$ and $q=2)$ at the stream $(\mathbf{A})$ and mesohabitat $(\mathbf{B})$ level. The shaded areas corresponded to $95 \%$ confidence intervals.

\section{TABLA 2}

Promedio por muestra y número total de géneros y abundancia de individuos encontrados en cada punto de estudio y clasificados en cada grupo funcional trófico (FFG)

TABLE 2

The average number of genders and abundance of individuals found in the studied streams and classified in each functional feeding group (FFG)

\begin{tabular}{lccc} 
& Géneros & Número de Individuos & Abundancia relativa (\%) \\
& & & 11.3 \\
Retirito & 43 & 1470 & 25.9 \\
La Lindosa & 50 & 3371 & 40.6 \\
Rincón de los Toros & 53 & 5290 & 22.3 \\
Tranquilandia & 38 & 2905 & 7.3 \\
Grupo Funcional Trófico (FFG) & 26 & 888 & 34.3 \\
$\quad$ Depredador & 3 & 14 & 2.0 \\
Filtrador & 6 & 518 & 15.8 \\
Fragmentador & 10 & 1399 & 25.7 \\
Raspador & 21 & 6440 & 0.2 \\
Recolector & 6 & 24 & 14.8 \\
Sin información & 17 & 3753 & \\
Hábitos variables & & & \\
\hline
\end{tabular}


escalas espaciales de estudio. A escala regional (Fig. 2A), los cuatro arroyos presentaron comunidades con grados de dominancia similares, siendo La Lindosa y el Rincón de los Toros los arroyos con mayor riqueza de taxones. Dentro de cada río (Fig. 2B), la roca madre presentó el mayor número de géneros, pero según lo observado en $\mathrm{q}=1$ y q $=2$, sólo algunos géneros concentran la mayor abundancia. Otros sustratos como la hojarasca y las macrófitas, tienen estimaciones de riqueza altas, pero sin marcadas diferencias entre $\mathrm{q}=0, \mathrm{q}=1 \mathrm{y} \mathrm{q}=2$.

De acuerdo al ANOSIM, la composición de las comunidades fue distinta entre sustratos $(\mathrm{R}=0.210, \mathrm{p}=0.007)$ y entre ríos $(\mathrm{R}=0.439$, $\mathrm{p}=0.001)$. Asimismo, la ordenación del NMDS (Fig. 3A) mostró una separación clara entre arroyos, dentro de los cuales a su vez se evidencia mayor cercanía entre las muestras tomadas en el mismo sustrato, especialmente de aquellas que corresponden a roca madre. En relación a los FFG, el ANOSIM mostró la existencia de diferencias entre ríos $(\mathrm{R}=0.285, \mathrm{p}=0.001)$ pero no entre los tipos de sustrato $(\mathrm{R}=0.100, \mathrm{p}$ $=0.072$ ). Esto se refleja en el NMDS (Fig. 3B), donde si bien la Lindosa parece separarse del resto de los arroyos, no se observan diferencias entre los distintos tipos de sustratos en términos de la composición de la comunidad.

Variaciones temporales en la diversidad: Se observaron cambios en la diversidad alfa y beta a lo largo de los años y los meses en el punto de estudio. Dentro de los ocho periodos evaluados (Fig. 4A), la mayor riqueza de géneros ocurrió durante el período de menor precipitación (enero 2017), mientras que los menores valores se observaron en la época de mayores precipitaciones (febrero-abril). En general, la diversidad beta entre muestreos fue alta (Fig. 4B). Entre 2014 y 2015 los procesos de recambio determinaron mayormente la diversidad beta observada, mientras que entre el 2015 y 2017 y a lo largo del 2017 el anidamiento fue el componente predominante en la diversidad beta.

\section{DISCUSIÓN}

La caracterización taxonómica mostró que la zona de estudio tiene una alta diversidad. La riqueza observada (89 géneros) fue mayor a la reportada para la Serranía de la Macarena (74 géneros), el río Bita (87 géneros) y otros ecosistemas fluviales en la Guayana venezolana (ver Mora-Day \& Blanco-Belmonte, 2008; Pereira, Garcia, Marcano, Lasso-Alcalá, \& Martínez-Escarbassiere, 2006; Souki et al., 2015). Asimismo, se observó que en la zona confluyen elementos de distintas regiones biogeográficas. Algunos de los géneros reportados en este trabajo han sido anteriormente asociados a la región de la Guayana (e.g. Scaphydra (Laython, 2017)), mientras que otros son de distribución andina (e.g. Parametriocnemus (Acosta \& Prat, 2010)) o amazónica (e.g. Leptohyphodes o Tricorythopsis (Domínguez, Zúñiga, \& Molineri, 2002)).

En cuanto a los cuatro nuevos registros para el país, se encontraron las siguientes consideraciones en su distribución geográfica. Se registra un nuevo género de la familia Elmidae: Luchoelmis. Este registro representa una importante ampliación de su distribución ya que esta estaba restringida al sur del continente, particularmente en Argentina, Chile y Bolivia (Archangelsky \& Brand, 2014). Con relación a los nuevos registros de la familia Chironomidae, Macropelopia es un género cuya distribución reportada es predominantemente Paleártica (Cranston \& Epler, 2013), no obstante, también ha sido reportado en Cuba (Bello González, Spies \& Téllez Martínez, 2013) y en Brasil (Rosa, Dias-Silva, \& Alvez, 2013), lo que sugería que una distribución más amplia. En el caso de Phaenopsectra, este sí había sido reportado previamente en Brasil y en la región andina (Acosta \& Prat, 2010; Henriques-Oliveira, Nessiman \& Dorvillé, 2003), por lo que su distribución se amplía a la región orinocense. Finalmente, el género Mayatrichia, del orden Trichoptera, ha sido reportado principalmente en norte y centro américa y una especie, M. illobia, en el sur del Ecuador (Holzenthal \& Calor, 2017). 


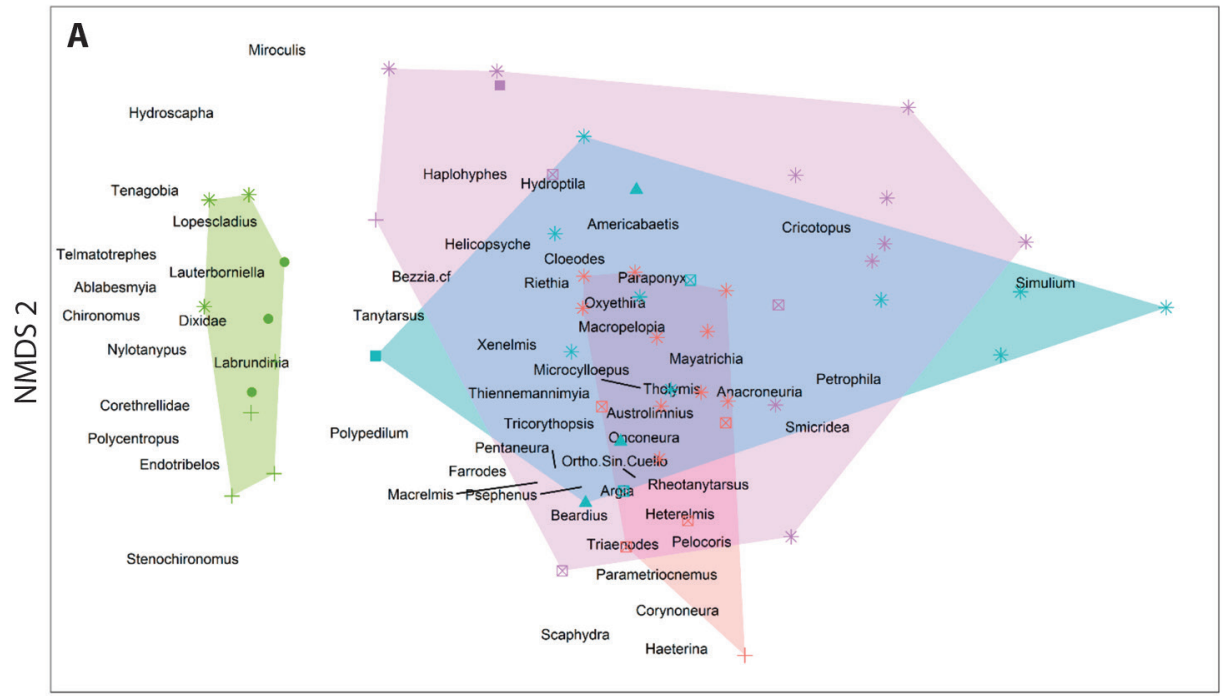

NMDS 1

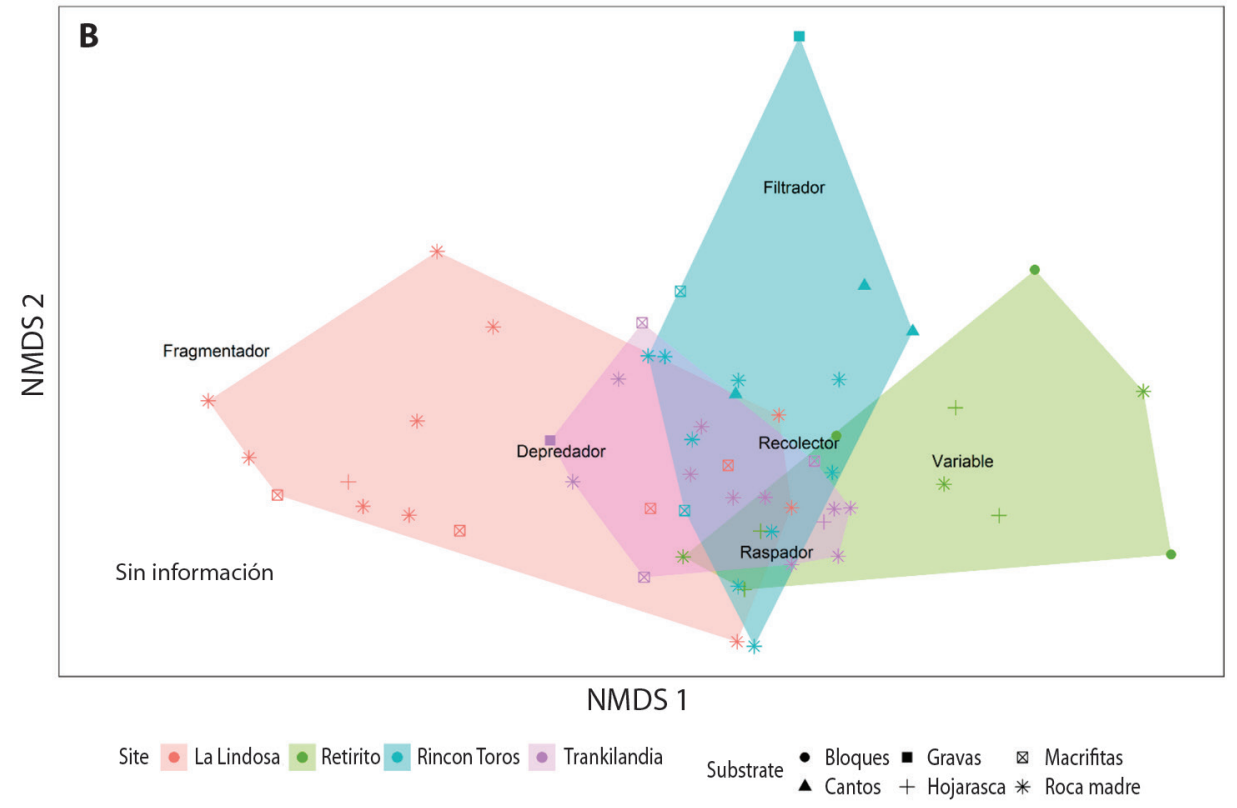

Fig. 3. (A) Ordenación (NMDS) de las muestras de acuerdo a la composición de géneros de la comunidad de macroinvertebrados acuáticos. Distancia: Morisita-Horn. Estrés de la ordenación: 0.19. Ajuste no métrico, $\mathrm{R}^{2}=0.968$. (B) Ordenación (NMDS) de las muestras de acuerdo a la clasificación en FFG de la comunidad de artrópodos acuáticos. Estrés de la ordeación: 0.13. Ajuste no métrico, $\mathrm{R}^{2}=0.984$.

Fig. 3. (A) Ordination (NMDS) of the samples according to the composition of genera of the aquatic invertebrate community. Distance: Morisita-Horn. Ordination stress: 0.18. Non-metric adjustment, $\mathrm{R}^{2}=0.968$. (B) Ordination (NMDS) of the samples according to the FFG classification of the aquatic invertebrate community. Ordination stress: 0.13 . Nonmetric adjustment, $\mathrm{R}^{2}=0.984$. 

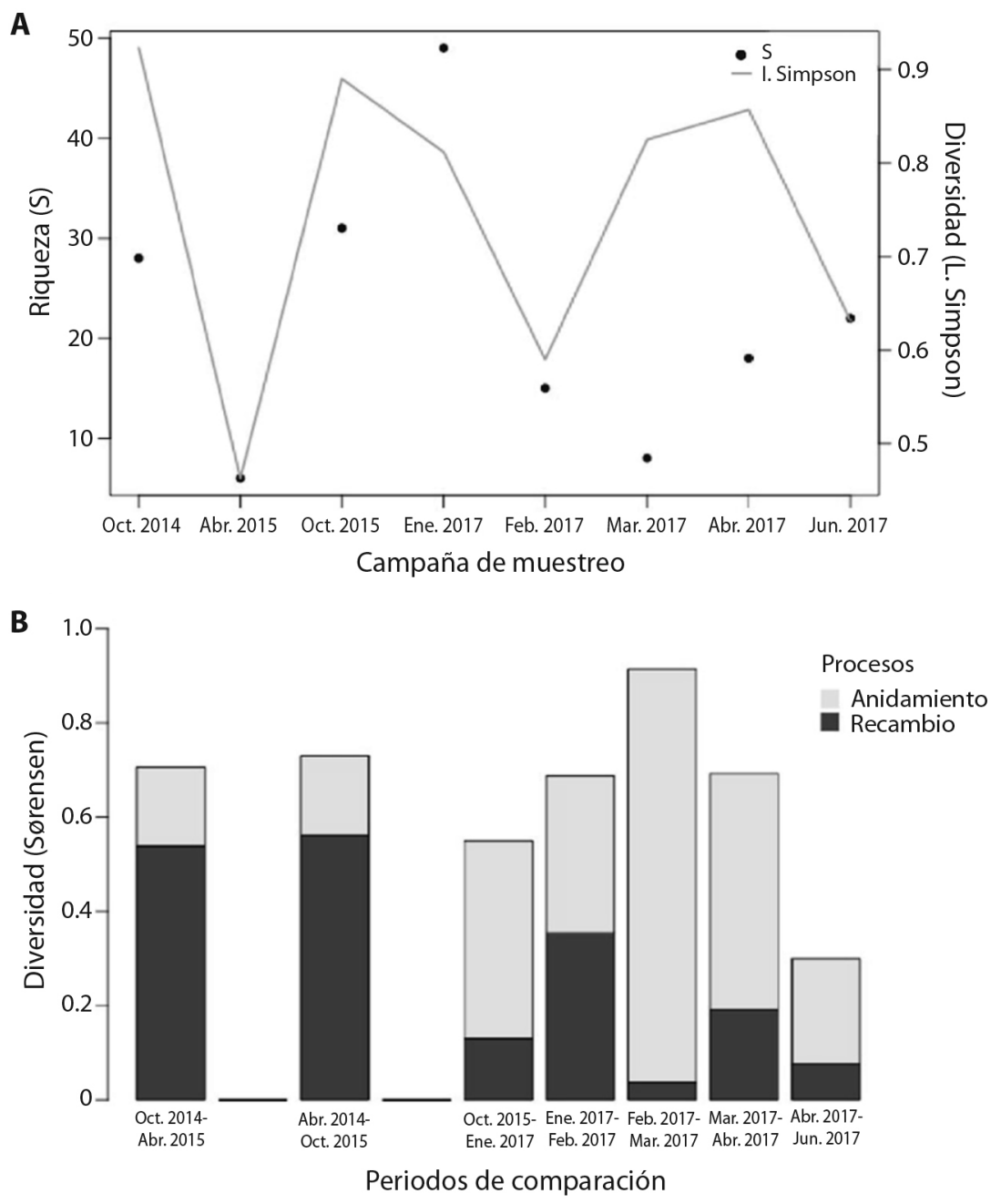

Fig. 4. Cambios temporales de la comunidad de macroinvertebrados en el caño Tranquilandia en términos de la diversidad alfa por campaña de muestreo (A), y de la diversidad Beta entre campañas (B). La diversidad Beta fue descompuesta en términos de los procesos de recambio y anidamiento.

Fig. 4. Seasonal changes of the macroinvertebrate community in the Tranquilandia stream measured as alpha diversity per sampling campaign (A), and Beta diversity between campaigns (B). Beta diversity was partitioned in terms of turnover and nestedness.

En lo que respecta a la caracterización trófica, esta primera aproximación mostró afinidad con la hipótesis de que los fragmentadores no son un grupo muy representado en la región tropical (e.g. Dobson, Magana, Mathooko, \& Ndegwa, 2002). No obstante, es una afirmación que merece ser estudiada en un futuro ya que, si bien la información secundaria consultada proviene principalmente de estudios Neotropicales, muchos de los géneros encontrados pueden tener una alta variabilidad intra- e interespecífica de su dieta (Tomanova, Goitia, \& Helešic, 2006; Boyero, Ramírez, Dudgeon, \& Pearson, 2009). Estas descripciones de la dieta deben asimismo ir acompañadas de un estudio más profundo de la morfología 
y el comportamiento de los organismos, con el fin de asignar los FFG de una manera más precisa (Ramírez \& Gutiérrez-Fonseca, 2014).

A pesar de la cercanía geográfica de los puntos de estudio y de sus aparentes características regionales similares (p.e. predominancia de roca madre, bajas conductividades, entre otras), se observó una alta variabilidad en la composición de la comunidad al comparar entre ríos. Esto podría estar asociado tanto a la baja capacidad dispersión de los taxones como a la influencia de características ambientales a pequeña escala (p.e. a escala de mesohábitat). Las restricciones a la dispersión son un factor relevante para explicar la alta disimilitud entre ríos que pertenecen a una misma red drenaje (Tonkin et al., 2018). Sin embargo, la falta de información acerca de la dispersión de las especies no nos permite evaluar el rol de este proceso en los patrones observados. Estudios futuros podrían ahondar en las habilidades de dispersión de las especies y cómo afectan los patrones de diversidad a escala regional.

Por otra parte, la variabilidad entre mesohábitats de un mismo río sugiere que la composición de la comunidad podría estar determinada por factores que actúan a pequeña escala. En particular, cambios en la diversidad alfa y en la composición de taxones indican que el tipo de mesohábitat podría ser determinante. Los mesohábitats tienen condiciones únicas en términos de tipo de sustrato, recursos y estrés hidráulico (Pardo \& Armitage, 1997), y por tanto promueven la compartimentalización de la comunidad, donde cada taxón tiene alta afinidad por cierto tipo de mesohábitat. Esto ha sido descrito previamente en otros ríos Neotropicales (p.e. Silva et al., 2014; González-Truji1lo, 2016) y apoya la idea de que los muestreos multihábitat son necesarios para caracterizar adecuadamente la diversidad a escala local y regional, como ha sido sugerido en otras regiones del mundo (e.g. Pardo \& Armitage, 1997).

Encontrar que la variación en la composición de los FFG sea significativa a escala regional pero no con respecto a los mesohábitats parece ser contradictorio con los resultados obtenidos al analizar la composición taxonómica de la comunidad. De hecho, difiere de lo registrado por otros estudios donde se ha establecido una fuerte relación entre tipos sustratos y grupos funcionales alimenticios (Demars, Kemp, Friberg, Usseglio-Polatera, \& Harper, 2012). Este resultado podría indicar que otras características diferentes al tipo de recurso (característica principal para definir mesohábitats en este estudio) tienen una mayor influencia sobre la presencia o ausencia de los grupos funcionales. Esto se soporta en otros estudios que han encontrado que las condiciones hidraúlicas podrían ser casi o más importantes que el tipo de recurso (Beisel et al., 1998; González-Trujillo, 2016; Silva et al., 2014; Tomanova et al., 2006).

La predominancia del anidamiento a lo largo del año 2017 parece indicar que existió una fuerte influencia de filtros ambientales relacionados con la estacionalidad (Baselga, 2010). Esto en conjunto con la disminución en la riqueza durante los meses de mayor cambio en el volumen de precipitaciones (febrero y marzo), sugieren que el anidamiento podría estar causado por una pérdida de especies a causa del aumento de eventos de creciente.

Los procesos de recambio en los meses de octubre a abril, por otra parte, podrían estar dados por cambios en la disponibilidad de los sustratos asociados también a la estacionalidad. La proliferación de las macrófitas ( $R$. clavijera) durante los meses más lluviosos, que actúan como sustrato de colonización y refugio ante los aumentos en el caudal (Pardo \& Armitage, 1997, Huttunen et al., 2017), podrían promover el establecimiento de nuevo taxones. Como lo demuestran los resultados, los parches de $R$. clavijera parecen tener una comunidad con una composición de taxones característica.

En conjunto, los resultados de este estudio demuestran que los ríos de la Serranía de la Lindosa albergan una alta diversidad de macroinvertebrados acuáticos. La estacionalidad y las diferencias en la heterogeneidad del hábitat dentro de los ríos parecen determinar los patrones de diversidad observados en escalas temporales y espaciales. Profundizar en su estudio es necesario para entender el 
funcionamiento de estos ecosistemas y mejorar la habilidad para generar estrategias de manejo y conservación.

Declaración de ética: los autores declaran que todos están de acuerdo con esta publicación y que han hecho aportes que justifican su autoría; que no hay conflicto de interés de ningún tipo; y que han cumplido con todos los requisitos y procedimientos éticos y legales pertinentes. Todas las fuentes de financiamiento se detallan plena y claramente en la sección de agradecimientos. El respectivo documento legal firmado se encuentra en los archivos de la revista.

\section{AGRADECIMIENTOS}

A la Fundación para la Promoción de la Investigación y la Tecnología (FPIT) por financiar una de las salidas de campo a través del convenio 201809, Proyecto No. 4.163. A Lizeth Alonso y Juan Pablo Álvarez Silva por acompañarnos en los muestreos. A Miriam y Javier por acogernos como si fuera nuestra casa. A Ruilson y a Alexander Vallejo por permitirnos el acceso a uno de los puntos de muestreo. A Diego Velásquez por su colaboración en la mejora de la calidad de las gráficas y figuras mostradas.

\section{RESUMEN}

Introducción: Describir los patrones de biodiversidad y los mecanismos que los subyacen es indispensable para afrontar las desenfrenadas presiones sobre los ecosistemas de agua dulce en el trópico. Esto es particularmente relevante en regiones escasamente estudiadas como el escudo guayanés colombiano Objetivo: Describir la estructura taxonómica y funcional de las comunidades de macroinvertebrados, y evaluar su variación a lo largo de escalas espaciales y temporales Métodos: Las comunidades de macroinvertebrados de cuatro ríos fueron muestreadas durante la época seca del 2017 y 2018 . Adicionalmente, se evaluaron los patrones de diversidad dentro y entre los ríos y entre diferentes meses de muestreo en uno de los cursos hídricos. Los patrones de diversidad fueron evaluados mediante la comparación de los patrones entre y dentro de los ríos de la diversidad alfa (diferencias en la riqueza y abundancia de taxones entre comunidades) y de la diversidad beta (diferencias en la composición de taxones entre comunidades). La diversidad alfa fue estimada usando los números de Hill, mientras que la diversidad beta se estimó usando los índices de Sørensen y Morisita-Horn y comparada usando un análisis de similaridad (ANOSIM). Resultados: La composición de taxones fue distinta dentro (escala de mesohábitat) y entre los ríos (escala regional). Sin embargo, la composición de FFG cambió entre, pero no dentro de los ríos. La comparación de los números de Hill y de la composición de taxones sugiere que el tipo de mesohábitat es un factor determinante de la diversidad taxonómica pero no de la diversidad de FFG en distintas escalas. En la escala temporal, la diversidad alfa fue baja para los meses con altas precipitaciones (febrero-abril) y alta para la temporada seca (enero). La diversidad beta temporal fue alta para los periodos muestreados y los procesos de anidamiento fueron el componente más notable de la diversidad beta a lo largo del año. Conclusión: Los ríos de la Serranía de la Lindosa albergan una alta diversidad de macroinvertebrados acuáticos. La estacionalidad y las diferencias en la heterogeneidad del hábitat dentro de los ríos parecen determinar los patrones de diversidad observados a diferentes escalas temporales y espaciales. Profundizar en su estudio es necesario para entender el funcionamiento de estos ecosistemas y mejorar la habilidad para generar estrategias de manejo y conservación.

Palabras clave: diversidad regional, características del hábitat, grupos funcionales tróficos, escala, Orinoco, Neotropical.

\section{REFERENCIAS}

Acosta, R., \& Prat, N. (2010). Chironomid assemblages in high altitude streams of the Andean region of Peru. Fundamental and Applied Limnology, 177(1), 57-79.

Archangelsky, M., \& Brand, C. (2014). A new species of Luchoelmis Spangler and Staines (Coleoptera: Elmidae) from Argentina and its probable larva. Zootaxa, 3779(5), 563-572.

Atuesta-Ibargüen, D.J. (2019). Composición florística y formas de vida de las macrófitas acuáticas de la serranía de La Lindosa (Guaviare), Guayana colombiana. Caldasia, 41(2), 301-312.

Baptista, D.F., Buss, D.F., Dias, L.G., Nessimian, J.L., Da Silva, E.R., De Moraes Neto, A.H.A., ... Andrade, L.R. (2006). Functional feeding groups of Brazilian Ephemeroptera nymphs: Ultrastructure of mouthparts. Annales de Limnologie, 42(2), 87-96.

Barker, J.E., Hutchens, J.J., \& Luken, J.O. (2014). Macroinvertebrates associated with water hyacinth roots and a root analog. Freshwater Science, 33(1), 159-167. 
Baselga, A. (2010). Partitioning the turnover and nestedness components of beta diversity. Global Ecology and Biogeography, 19(1), 134-143.

Baselga, A., Orme, D., Villeger, S., De Bortoli, J., \& Leprieur, F. (2018). betapart: Partitioning Beta Diversity into Turnover and Nestedness Components. Retrieved from https://cran.r-project.org/ package $=$ betapart

Beisel, J., Usseglio-Polatera, P., Thomas, S., \& Moreteau, J. (1998). Stream community structure in relation to spatial variation: the influence of mesohabitat characteristics. Hydrobiologia, 389, 73-88.

Bello González, O.C., Spies, M., \& Téllez Martínez, B. (2013). Estado del conocimiento de la familia Chironomidae (Insecta: Diptera ) en Cuba. Dugesiana, 20(2), 233-242.

Boyero, L., Ramírez, A., Dudgeon, D., \& Pearson, R.G. (2009). Are tropical streams really different? Journal of the North American Benthological Society, 28(2), 397-403.

Cárdenas López, D., Castaño Arboleda, N., Zubieta Vega, M., \& Jaramillo Echeverry, M. (2008). Flora de las formaciones rocosas de la Serranía de La Lindosa. Bogotá, Colombia: Instituto Amazónico de Investigaciones Científicas -Sinchi-.

Ceneviva-Bastos, M., Prates, D.B., de Mei Romero, R., Bispo, P.C., \& Casatti, L. (2017). Trophic guilds of EPT (Ephemeroptera, Plecoptera, and Trichoptera) in three basins of the Brazilian Savanna. Limnologica, $63,11-17$.

Chao, A., Gotelli, N.J., Hsieh, T.C., Sander, E.L., Ma, K.H., Colwell, R.K., \& Ellison, A.M. (2014). Rarefaction and extrapolation with Hill numbers: A framework for sampling and estimation in species diversity studies. Ecological Monographs, 84(1), 45-67.

Chao, A., \& Jost, L. (2012). Coverage-based rarefaction and extrapolation: Standardizing samples by completeness rather than size. Ecology, 93(12), 2533-2547.

Chará-Serna, A.M., Chará, J.D., Zúñiga, M.D.C., Pedraza G.X., \& Giraldo, L.P. (2010). Clasificación trófica de insectos acuáticos en ocho quebradas protegidas de la ecorregión cafetera colombiana. Universitas Scientiarum, 15(1), 27-36.

Chará-Serna, A.M., Chará, J.D., Zúñiga, M.C., Pearson, R.G., \& Boyero, L. (2012). Diets of leaf litter-associated invertebrates in three tropical streams. Annales de Limnologie - International Journal of Limnology, 48(2), 139-144.

Chaves-Ulloa, R., Umaña-Villalobos, G., \& Springer, M. (2014). Downstream effects of hydropower production on aquatic macroinvertebrate assemblages in two rivers in Costa Rica. Revista de Biología Tropical, $62,179-201$
Clarke, K.R. (1993). Non-parametric multivariate analyses of changes in community structure. Australian Journal of Ecology, 18(1), 117-143.

Collins, P.A., \& Paggi, J.C. (1997). Feeding ecology of Macrobrachium borelli (Nobili) (Decapoda: Palaemonidae) in the flood valley of the River Paraná, Argentina. Hydrobiologia, 362(1-3), 21-30.

Cranston, P.S., \& Epler, J.H. (2013). The larvae of Tanypodinae (Diptera: Chironomidae) of the Holarctic region-Keys and diagnoses. Insect Systematics and Evolution Supplements, 66, 39-136.

Cummins, K.W. (1973). Trophic relations of aquatic insects. Annual Review of Entomology, 18(220), 183-206.

da Silva Laurindo, F., Calcidoni Moreira, D., Lucas Bochini, G., \& Silveira Ruiz, S. (2008). Hábitos alimentares de larvas de Chironomidae (Insecta: Diptera) do córrego Vargem Limpa, Bauru, SP, Brasil. Biotemas, 21(2), 155-159.

Demars, B.O.L., Kemp, J.L., Friberg, N., Usseglio-Polatera, P., \& Harper, D.M. (2012). Linking biotopes to invertebrates in rivers: Biological traits, taxonomic composition and diversity. Ecological Indicators, 23, 301-311.

Dobson, M., Magana, A., Mathooko, J.M., \& Ndegwa, F.K. (2002). Detritivores in Kenyan highland streams: More evidence for the paucity of shredders in the tropics? Freshwater Biology, 47(5), 909-919.

Domínguez, E., \& Fernández, H.R. (2009). Macroinvertebrados bentónicos sudamericanos. Sistemática y biología (1 ${ }^{\text {ra }}$ Ed.). Tucumán, Argentina: Fundación Miguel Lillo.

Domínguez, E., Zúñiga, M.C., \& Molineri, C. (2002). Estado actual del conocimiento y distribución del orden Ephemeroptera (Insecta) en la región amazónica. Caldasia, 24(2), 459-469.

Elosegi, A., \& Sabater, S. (2009). Conceptos y técnicas en ecología fluvial. Bilbao, España: Fundación BBVA.

Fogaça, F.N.O., Gomes, L.C., \& Higuti, J. (2013). Percentage of Impervious Surface Soil as Indicator of Urbanization Impacts in Neotropical Aquatic Insects. Neotropical Entomology, 42(5), 483-491.

Gabbud, C., Robinson, C.T., \& Lane, S.N. (2019). Subbasin and temporal variability of macroinvertebrate assemblages in Alpine streams: when and where to sample? Hydrobiologia, 830(1), 179-200.

Gallegos Sánchez, S.A. (2013). Effect of riparian vegetation cover and season on aquatic macroinvertebrate assemblages in the Ecuadorian Andes (Master Thesis). Norwegian University of Life Sciences, Noruega. 
Giraldo-Cañas, D. (2001). Relaciones fitogeográficas de las sierras y afloramientos rocosos de la Guayana colombiana: un estudio preliminar. Revista Chilena de Historia Natural, 74, 353-364.

González-Trujillo, J.D. (2016). Trait-based responses of caddisfly assemblages to the partial channelization of a High-Andean stream. Hydrobiologia, 766(1), 381-392.

Granados-Martínez, C., \& Batista, A. (2017). Macroinvertebrados acuáticos. In C.A. Lasso \& M.A. MoralesBetancourt (Eds.), III. Fauna De Caño Cristales, sierra de La Macarena, Meta, Colombia (pp. 47-65). Bogotá, Colombia: Insituto de Investigación de Recursos Biológicos Alexander von Humboldt.

Granados-Martínez, C., Lasso, C.A., Núñez-Avellaneda, M., \& Morales-Betancourt, M.A. (2018). Macroinvertebrados acuáticos de los ríos Guayabero medio, bajo Losada y bajo Duda, Sierra de La Macarena, Meta, Colombia. In C.A. Lasso, M.A. MoralesBetancourt, \& I.D. Escobar-Martínez (Eds.), V. Biodiversidad de la sierra de La Macarena, Meta, Colombia (Parte I, pp. 97-120). Bogotá, Colombia: Instituto de Investigación de Recursos Biológicos Alexander von Humboldt.

Granados-Martínez, C., \& Montoya, D. (2017). Macroinvertebrados acuáticos. In F. Trujillo \& C.A. Lasso (Eds.), IV. Biodiversidad del rio Bita, Vichada, Colombia (pp. 121-141). Bogotá, Colombia: Instituto de Investigación de Recursos Biológicos Alexander von Humboldt.

Hammond, D.S. (2005). Tropical forests of the Guiana Shield: Ancient forests of the modern world. Cambridge, MA: CABI Publishing.

Heino, J. (2008). Patterns of functional biodiversity and function-environment relationships in lake littoral macroinvertebrates. Limnology and Oceanography, 53(4), 1446-1455.

Henriques-Oliveira, A.L., Nessimian, J.L., \& Dorvillé, L.F. (2003). Feeding habits of chironomid larvae (Insecta: Diptera) from a stream in the Floresta da Tijuca, Rio de Janeiro, Brazil. Revista Brasleira de Biologia, 63(2), 269-281.

Holzenthal, R.W., \& Calor, A.R. (2017). Catalog of the Neotropical Trichoptera (Caddisflies). ZooKeys, 654, $1-566$.

Horn, H.S. (1966). Measurement of "overlap" in comparative ecological studies. The American Naturalist, 100(914), 419-424.

Hsieh, T.C., Ma, K.H., \& Chao, A. (2016). iNEXT: an R package for rarefaction and extrapolation of species diversity (Hill numbers). Methods in Ecology and Evolution, 7(12), 1451-1456.
Huttunen, K.L., Mykrä, H., Oksanen, J., Astorga, A. Paavola, R., \& Muotka, T. (2017). Habitat connectivity and in-stream vegetation control temporal variability of benthic invertebrate communities. Scientific Reports, 7(1), 1-9. https://doi.org/10.1038/ s41598-017-00550-9

Kruskal, J.B. (1964). Multidimensional scaling by optimizing goodness of fit to a nonmetric hypothesis. Psychometrika, 29(1), 1-27.

Lasso, C.A., \& Morales-Betancourt, M.A. (2017). III. Fauna De Caño Cristales, sierra de La Macarena, Meta, Colombia. Bogotá, Colombia: Instituto de Investigación de Recursos Biológicos Alexander von Humboldt.

Lasso, C.A., Morales-Betancourt, M.A., \& Escobar-Martínez, I.D. (2019). Biodiversidad de la sierra de La Macarena, Meta, Colombia. Ríos Guayabero medio, bajo Losada y bajo Duda (Parte I). Bogotá, Colombia: Instituto de Investigación de Recursos Biológicos Alexander von Humboldt.

Laython, M. (2017). Los Coleópteros Acuáticos (Coleoptera: Insecta) en Colombia. Distribución y Taxonomía (Master Thesis). Universidad Nacional de Colombia, Bogotá, Colombia.

Ligeiro, R., Melo, A.S., \& Callisto, M. (2010). Spatial scale and the diversity of macroinvertebrates in a Neotropical catchment. Freshwater Biology, 55(2), 424-435.

Mendes, F., Kiffer, W.P., \& Moretti, M.S. (2017). Structural and functional composition of invertebrate communities associated with leaf patches in forest streams: a comparison between mesohabitats and catchments. Hydrobiologia, 800(1), 115-127.

Merritt, R.W., Dadd, R.H., \& Walker, E.D. (1992). Feeding behavior, natural Food, and nutritional relationships of larval mosquitos. Annual Review of Entomology, 37(1), 349-376.

Merritt, R.W., Cummins, K.W., \& Berg, M.B. (2008). An introduction to the aquatic insects of North America ( $4^{\text {th }}$ Ed.). Dubuque: Kendall/Hunt Publising Co.

Mihuc, T., \& Toetz, D. (1994). Determination of Diets of Alpine Aquatic Insects Using Stable Isotopes and Gut Analysis. The American Midland Naturalist, 131(1), 146-155.

Mora-Day, J., \& Blanco-Belmonte, L. (2008). Macroinvertebrados acuáticos del alto río Paragua, cuenca del río Caroní, Estado Bolívar, Venezuela. In J.C. Señaris, C.A. Lasso, \& A.L. Flores (Eds.), Evaluación Rápida de la Biodiversidad de los Ecosistemas Acuáticos de la Cuenca Alta del Río Paragua, Estado Bolivar, Venezuela. RAP (pp. 97-109). Arlington, VA, USA: Conservation International.

Oksanen, J., Blanchet, F.G., Friendly, M., Kindt, R., Legendre, P., McGlinn, D., ... Wagner, H. (2017). vegan: 
Community Ecology Package. Retrieved from https:// cran.r-project.org/package= $=$ vegan

Pardo, I., \& Armitage, P.D. (1997). Species assemblages as descriptors of mesohabitats. Hydrobiologia, 344(13), 111-128.

Pereira, G., Garcia, J.V., Marcano, A., Lasso-Alcalá, O.M., \& Martínez-Escarbassiere, R. (2006). Los macroinvertebrados bentónicos de la confluencia de los ríos Orinoco y Ventuari. In C. A. Lasso, J.C. Señaris, L.E. Alonso, \& A.L. Flores (Eds.), Evaluación Rápida de la Biodiversidad de los Ecosistemas Acuáticos en la Confluencia de los ríos Orinoco y Ventuari, Estado Amazonas (Venezuela). Boletín RAP de Evaluación Biológica 30 (1 $1^{\text {st }}$ Ed., pp. 96-106). Washington, DC. USA.: Conservation International.

Prat, N., González-Trujillo, J.D., \& Ospina-Torres, R (2014). Clave para la determinación de exuvias pupales de los quironómidos (Diptera: Chironomidae) de ríos altoandinos tropicales. Revista de Biología Tropical, 62(4), 1385-1406.

Ramírez, A., \& Gutiérrez-Fonseca, P.E. (2014). Functional feeding groups of aquatic insect families in Latin America: A critical analysis and review of existing literature. Revista de Biología Tropical, 62(2), 155-167.

Rosa, B.F.J.V., Dias-Silva, M.V.D., \& Alves, R.G. (2013). Composition and Structure of the Chironomidae (Insecta: Diptera) Community Associated with Bryophytes in a First-Order Stream in the Atlantic Forest, Brazil. Neotropical Entomology, 42(1), 15-21.

Salerno, P.E., Ron, S.R., Señaris, J.C., Rojas-Runjaic, F.J.M., Noonan, B.P., \& Cannatella, D.C. (2012) Ancient tepui summits harbor young rather than old lineages of endemic frogs. Evolution, 66(10), 3000-3013.

Santos, I.G. (2014). Colonização de macroinvertebrados bentônicos em detritos foliares em riacho de primeira ordem da reserva biológica de Saltinho-Pernambuco (Master Thesis). Universidade Federal de Pernambuco, Recife, Brasil.

Saulino, H., Corbi, J., \& Trivinho-Strixino, S. (2014). Aquatic insect community structure under the influence of small dams in a stream of the MogiGuaçu river basin, State of São Paulo. Brazilian Journal of Biology, 74(1), 79-88.

Sganga, J.V. (2011). Variabilidad espacial y estructura de las comunidades de Trichoptera (Insecta) en arroyos del Parque Provincial Salto Encantado del Valle del Cuñá-Pirú (Misiones, Argentina) (Doctoral dissertation). Universidad de Buenos Aires, Buenos Aires, Argentina.

Silva, D.R.O., Ligeiro, R., Hughes, R.M., \& Callisto, M. (2014). Visually determined stream mesohabitats influence benthic macroinvertebrate assessments in headwater streams. Environmental Monitoring and Assessment, 186(9), 5479-5488.

Souki, M.E, Blanco-Belmonte, L., Lasso, C.A., Mora-Day, J., Magalhaes, C., Pisapia, D., ... Lasso-Alcalá, O.M. (2015). Composición y distribución de la comunidad de insectos acuáticos en un gradiente espacial del alto río Cuyuní, Guayana venezolana. Memoria de la Fundación La Salle de Ciencias Naturales, 71, 79-103.

Spinelli, G.R., \& Wirth, W.W. (1991). The Neotropical Predaceous Midges of the genus Bezzia (Diptera: Ceratopogonidae) Part IV. The dentifemur and venustula Groups. Insecta Mundi, 5(1), 1-18.

Tomanova, S., Goitia, E., \& Helešic, J. (2006). Trophic levels and functional feeding groups of macroinvertebrates in neotropical streams. Hydrobiologia, 556(1), 251-264.

Tonkin, J.D., Altermatt, F., Finn, D.S., Heino, J., Olden, J.D., Pauls, S.U., \& Lytle, D.A. (2018). The role of dispersal in river network metacommunities: Patterns, processes, and pathways. Freshwater Biology, 63(1), 141-163.

Trivinho-Strixino, S. (2011). Larvas de Chironomidae: Guia de identificação. São Carlos, Brasil: Depto Hidrobiologia/Lab. Entomologia Aquática/UFSCar.

Wiederholm, T. (1983). Chironomidae of the Holarctic region Keys and diagnoses. Part I - Larvae. Entomologica Scandinavica, 19, 1-457. 